\title{
Research on the Development and Cultural Orientation of Chinese Pre-Qin Hemp Textile Industry
}

\author{
$\operatorname{Lin~} \mathrm{Li}^{1}$ \\ ${ }^{1}$ Jilin Engineering Normal University, Jilin, Changchun, China, 130052
}

Keywords: hemp textile industry; Pre-Qin Period; production; culture orientation

\begin{abstract}
Hemp textile plays a very important role in the life of ancient Chinese people and plays a very important role in the origin of Chinese civilization and the development of the times. Chinese hemp textile industry has a long history, raw material cultivation and processing and textile technology have been continuously improved, and the scale of production has been continuously expanding. It has ushered in the first peak of development during the Spring and Autumn and Warring States Period. Hemp is one of the earliest textile fabrics in China and has a practical function of keeping body warm. The level of production and processing technology determines the difference in the thickness of the linen fabric, but the factors such as hierarchy, ethics, value standards and hemp fabrics are combined to give unique cultural connotations for linen fabrics.
\end{abstract}

\section{Introduction}

Chinese hemp textile industry has a long history. Burlap and burlap were the main clothing fabrics of the pre-Qin period until they were gradually replaced by cotton cloth during the Song and Ming dynasties. A lot of historical data confirms that the ancient Chinese hemp textile industry has reached a considerable level. Hemp fabrics play a very important role in the life of ancient Chinese people and played a major role in the origin of the Chinese civilization and the development of the times. Especially during the Spring and Autumn Period and the Warring States Period of the Pre-Qin period, people used silk and hemp together and used hemp as a raw material to carry out textile production. The production and processing and post-processing were more elaborate and systematic. The development of Chinese first hemp textile industry peaked.

\section{The Long History of Chinese Hemp Textile Industry}

Chinese hemp textiles are more ancient than silk fabrics. Since the distant Neolithic era, people have learned how to use hemp and kudzu fibers to make twine and linen for people to use. In many sites in the Neolithic Age, linen tools were unearthed, and the prints of linen fabrics were also found. Some pottery vessels in the Yangshao Culture, Dawenkou Culture, and Longshan Cultural Site in the Yellow River Basin often have clear linen prints. The original spinning wheel was found in the Hemudu site in Yuyao, Zhejiang, about 7,000 years ago. Three hemp ropes and two hemp ropes were found at the site. In the ruins of Caoheshan Mountain in Wu County, Jiangsu, there are three pieces of precious Gap fragments dating back to 6,000 years ago. The three pieces of Gebbu debris are double-stranded ribbed gebra cloth. The diameter (projection) of the double-stranded yarn is $0.45-0.90 \mathrm{~mm}$, and the twisting direction is S. The warp density is approximately 10 per $\mathrm{cm}$, and the density of the weft is $13 \mathrm{~cm} / \mathrm{cm}$. -14 roots, about 26-28 ribs [1].

From the Yangshao culture to the Longshan cultural era, the Ma textile industry has seen tremendous progress. Although linen weaving is still a plain weave method, the thickness of the latitude and longitude lines is very different, and the texture is even more detailed than the Yangshao period. For example, the wrapping cloth on the bronze bell at the Taosi site in Shanxi Province has $16 \times 22$ square cm; the sackcloth on the Qijia culture clay pot in Dahezhuang, Yongqing, Gansu province is $30 \times 30 \mathrm{~cm} 2$; Zhejiang Wuxing money The sackcloth unearthed at the foothills site is $24 \times 24$ or $31 \times 20$ square centimeters, and the diameter of each twine is less than 0.5 millimeter. This shows that during the Longshan Culture, Geb's textile technology was even higher. 
The hemp textiles of the Xia and Shang Dynasties have been quite elegant. The hemp fabrics of the Xia and Shang Dynasties were found in the Taizhou West Site of Hebei Province and Yin Ruins of Anyang County. The two pieces of hemp fiber fabric found in the early Shang Dynasty sites in the Xicheng Village of Artemisia City were plain weave. One of the warp yarns had a density of $14-16 / \mathrm{cm}$, and the weft was $9-10 / \mathrm{cm}$; the other warp was $18-20$. The root $/ \mathrm{cm}$, the weft yarn is $6-8$ pieces/cm, the warp yarn projection width is only $0.8-1 \mathrm{~mm}$, the weft yarn is $0.41 \mathrm{~mm}$, and its technical level is comparable to that of the linen excavated from the Mawangdui in West Han Dynasty [2].

In the Zhou Dynasty, the hemp textile technology became increasingly sophisticated, textile tools continued to improve, and spinning wheels were various. The fineness of hemp fabrics was comparable to that of silk. During the Spring, Autumn and Warring States period, the production level of hemp fabrics was even more impressive. In 1952, white burlap pieces were found in the Warring States Tomb at No. 406 Wulibei, Changsha, Hunan. The weft and weft densities of the fabrics were 280 per $10 \mathrm{~cm}$ warp yarns and 240 per $100 \mathrm{~cm}$ weft yarns. The leading fine cloth is woven at 254 per 100,000 square meters. 248) It is important to know that $3.4 \%$ of the fine fabrics can be known from the ancient Chinese textile technology."

\section{Production and Technological Innovation of Hemp Textile Industry in the Pre-Qin Period}

Hemp fabric materials are divided into two kinds of hemp and kudzu. The fibrous stalks in the stems of the genus Georgi belong to the perennial vine of the leguminous family. Its leaves resemble maple leaves. They are cyan in color, long and thin, and the flowers are purple in color. Hemp is a herbaceous plant with fibrous skins in the stems. It is the fibers of hemp fabric that are obtained after peeling and processing. It is particularly suitable for the growth of $\mathrm{Ma}$ and $\mathrm{Ge}$ in warm and warm climates in hills and valleys. In ancient China before the cotton was not popularized, the general public used hemp fabrics and ramie fabrics as clothing materials. People had long understood the cultivation of GE plants. In the Book of Songs, there are dozens of places to describe the cultivation and processing of Ge and Ma. In the ruins of the Linjia Majiayao in Dongxiang, Gansu Province in the late Neolithic Age, hemp seeds were found preserved in pots. This site dates back to 5000 BC. According to archeological findings, hemp has been a common textile raw material in the Shang Dynasty and it has been widely cultivated. The cannabis plantation in Shang Dynasty was inherited from the past. After long-term cultivation and provocation by ancient working people, it became an excellent raw material for textiles, laying a good foundation for the development of ancient Chinese hemp textile industry. The hemp fabric unearthed in the Han Tomb of Mawangdui No. 1 in Changsha, Hunan Province was examined and analyzed. The projection width of hemp fiber was 21.83 microns and the cross-sectional area was 153.11 square microns. Its variety is very good and it is close to the modern cultivation of marijuana. This is the result of long-term planting and breeding of improved varieties by the working people of our country [3].

Cannabis production before the Western Zhou Dynasty was most famous for the production of hemp in Henan and Shandong. In the article "Geng Gong”, the two states of Qing and Yu used tribute as tribute. "Historical Records and Biographies" also recorded that Qilu was rich in Sangma. The Huai River Yangtze River is suitable for the growth of ramie, and Chen Guo, located next to Yingshui in Huaibei, attaches equal importance to the cultivation of ramie and hemp. Far from the location of the Wu State on the southern bank of the Yangtze River, as early as the Liangxu Culture Period, Ramie was used as a raw material for textiles. Naturally, such crops will not be reduced during the Zhou Dynasty. Gehe grass is a wild plant. This kind of fiber is a natural source of artificial hemp and ramie insufficiency. Therefore, the raw material of the hemp textile industry in China was very abundant during the pre-Qin period.

As the size of the hemp textile industry continues to expand, Zhou Chao began to adopt the model of large-scale government-run workshops and small-scale handicraft workshops for the family in the management system of the hemp textile industry. The state has set up special management agencies for the production of silk, hemp, and silk from textile raw materials, the 
collection of dyes, and a series of production processes from spinning, weaving, dyeing, and garment manufacturing. "Pingge" is responsible for the collection of raw materials, "Principals" in charge of the distribution of raw materials to the production workshops, and the collection of hemp textiles of different grades on time for storage, for various consumption needs of the royal family or princes. Farmers' home textile weaving is also supervised by local administrative agencies. State power exercises the function of inspecting production, and the ruler gets more "clothing" products. It is not difficult to see that the ruling class of the Zhou dynasty paid equal attention to two kinds of textile production systems, treating them as mutually complementary forms of textile production and being the two main sources of national linen sources [4].

During the Spring and Autumn Period, Chinese textile industry, driven by the tide of social and economic changes, has a much larger scale of production than in the past. There are textile workshops inside and outside the capitals of the nations and in some other larger cities. Directly affiliated with the official or aristocratic textile weaving "women workers", under the direct supervision of officials such as "Women's Work" and "Sewing People", the labor time is longer and the labor intensity is greater. A small family of small handicraftsmen also produces hemp textiles in large quantities, and set up a "sell" market.

The Warring States Period was an important historical stage in the rapid development of Chinese ancient textile industry. With the unprecedented expansion of the number of individual smallholders during the Warring States Period, the small-farm economy developed by men and women has been rapidly developed. During the Warring States period, all feudal countries implemented the policy of support and protection for the small-scale family handicrafts. For example, the decree of merchants in the Qin State even stipulated clearly [5]: "The people who work hard to cultivate their own crops are the ones to recover." Not only do family textiles as an important part of "the industry", but also For smallholder farmers who have increased their production of agricultural products (millet) and handicraft products in the "business", they can also be exempt from service. During the Warring States period, the handicrafts of the government were weakened compared to the Spring and Autumn period of the Western Zhou Dynasty, but they still occupied a considerable proportion in the whole handicraft industry at that time. The government-run hemp textile industry was also the best textile talent in the world and created various new hemp textiles. The products, which were directly managed to satisfy the rulers' enjoyment of life and political and military needs, were an important part of the feudal society at that time.

In the long-term production process, the production technology of the hemp textile industry continues to progress and innovate. For example, in the degumming technology of hemp fabrics, the earliest people used stone tools to beat the hemp plants and soften them, and then they tore them into thin slugs, which were used to make ropes or braids into nets. Due to the presence of colloids, without proper degumming, hemp fibers can be relatively stiff. In the process of long-term contact with nature, humans find that the stems fall down in the leeches. Because of the soaking of water and the irradiation of sunlight, it is easier to peel, and the hemp fiber becomes flexible, slender, and white, so people are interested in Stems are soaked in water. This natural spotting method is the earliest method of degumming. People in the Yangtze River Valley in China started to use it in late Neolithic, and during the Shang and Zhou Dynasties they also began to prevail in the Yellow River Basin. The traces of degumming can be identified in the Qiangshan Neolithic site in Zhejiang Province and the linen pieces unearthed in Beijing Pinggu Shang Tomb. Around the Spring and Autumn Period, people began to cook sesame in ash. There are two types of gray matter: one is ash juice of eucalyptus wood, which is a soda ash material; the other is ash of shells and the like plus water, ie lime water. The ramie cloth unearthed in the spring and autumn tombs of Xi Gaoquan, Baoji, Shaanxi Province, was analyzed and analyzed. The fiber separation degree was good. It was scouring and degumming. It can be seen that there was already some experience in mastering the gray matter ratio and scouring time. The degumming technology of Chinese pre-Qin period was very effective. At present, in the ecological Xiabu production technology of the non-heritage catalog, the degumming of ramie still uses the method of bleaching the plant ash, lime water, and sun and dew [6]. 
Along with the continuous advancement of degumming technology, the technology of hemp and hemp weaving in Chinese pre-Qin period has also gradually improved. The degree of coarseness of the ancient Chinese linen was explained by "l". "L" is expressed by the number of warps on a linen fabric with a width of 2" (Hanban, which is today's $50 \mathrm{~cm}$ ). One of the 80 warp yarns is liter, 160 is two liters, and so on [7]. On the contrary, the lower the liters, the worse the quality of the fabric. Referring to this data, the finest litters in the Spring and Autumn period can often reach 30 liters, and its fineness is equivalent to today's poplin, which shows the superiority of the hemp textile technique during the pre-Qin period.

\section{Cultural Orientation of Chinese Pre-Qin Hemp Textile Industry}

Ma is one of the earliest textile fabrics in China. It has a practical function of keeping body warm. The level of production and processing technology determines the external coarseness of hemp fabrics, but the factors such as hierarchy, ethics, value standards and hemp fabrics when quality differences are combined, linen fabrics are given unique cultural connotations.

Xiaoqin Zhongxin is the essence of ancient Chinese culture. In the process of Chinese historical transformation, some ideas will disappear or be innovated and changed, but they can be consistent in maintaining respectability and differentiating distances. Hemp fabrics differentiate between rich and poor and class in a special way. As the choice of apparel and apparel fabrics was influenced by traditional Chinese concepts, hemp products had a deep shadow of the patriarchal patriarchal rites. In the pre-Qin period, people of different classes and different identities wore hemp fabrics, and the number of "up" was different. Seven to nine liters of linen fabric are used by slaves. Ten to fourteen liters of hemp fabric are used by civilians. Fifteen liters of linen fabric are comparable to silk fabrics. They are materials used by Dadi nobles. Thirty liters of fine hemp fabrics are even more expensive and delicate than silk, and can only be used by nobles and aristocrats. This also reflects the honorable position of the dresser [8].

In ancient Chinese funeral culture, hemp textiles carried another cultural connotation. Under the traditional etiquette of the utmost goodness and filial piety, hemp fabrics satisfy the human subjective expressions of their social, ideological and spiritual aspects with their unique fabric characteristics. The filial piety reflects the sorrow and dismay of the dead people with completely different cultural characteristics. The coarser the mourning, the more closely reflects the kinship of living people and the deceased, and the more uncomfortable the body feel, the more they can express the grief of the mourners, thus embodying the filial piety culture. The "three liters" in linen fabrics are the lowest ascending position. Generally, they don't do everyday clothes, but in mourning clothes, they are the most important thing. "Three liters" of filial piety is made of unripe raw ramie. The fabric is stiff, the fiber is sparse and contains a lot of hemp fiber impurities, and the comfort is particularly poor. It is the clothes worn by children as their parents and princes as emperors. Because the edge of the mourning service has not been closed, it is a form of fracture. According to historical records, when his father defends his filial piety, Zanzi leans on the grass and sleeps on his knees. He wears burlap clothes without seams, he has twine on his head and waist, and he wears straw shoes to live a hard life. Four liters, five liters, and six liters of hors d'oeuvre are mourning and rough, but they are better than the quality of the tumultuous and numbness, and the wearer's affection is farther than the "decline"; seven litres, eight liters, nine The literary mourning service is better than the seven liters of mourning, and the quality has been improved and the comfort has been improved. Ten liters, eleven liters, and twelve liters of mourning garments have been made of cooked hemp fibres, making them softer and more delicate. However, the mourning sentiment expressed to the deceased person has been reduced. As the rise rises, the increase in the quality of hemp fabrics also means that the family relationship is becoming increasingly alienated.

In the process of continuous development and improvement of the ancient Chinese textile industry, the innovation of Chinese civilization and technology interacted with each other. The ancient Chinese sages not only demonstrated the intimateness and inferiority of noble, noble, and blood relations through hemp textiles, but also embodied the deep ethical concepts such as virtue and filial piety. Even the dyeing process of the textile industry can even bring out the ancient society. 
As early as six or seven thousand years ago, the ancient Chinese could use hematite powder to dye the linen red. During the Shang and Zhou dynasties, court officials set up full-time bureaucrats to "smear grass" and “dyeing people” to manage dyeing production. After numerous explorations over a long period of time, during the Warring States period, two methods of "grass dyeing" (plant dyes) and "stone dyeing" (mineral dyes) were formed. The dyeing techniques have been continuously improved, and the dyed colors have been continuously increased to blue-dyed blue. The process is already very mature. Cyan is dyed indigo from bluegrass. Therefore, Xunzi said in the "Encouragement": "Green is blue and blue is blue". In order to dye silk jute into many different colors, not only one dye, then dye, three dyes, and even five After dyeing, dyeing and dyeing were dyed in seven dyeing techniques, Mozi sighed with "Mo Zi • Dyeing": White silk dyed in blue dyed water became blue and dyed in yellow dyed water became yellow The dyestuff that has entered has changed, and the color of the silk has changed. Five times into five different colors, silk becomes five colors. Mozi thus associates himself with the fact that many things in society are related to "dyeing." Admonish the emperors, princes, doctors, and scholars that they must correctly choose their own relatives and friends in order to obtain good influence and positive influence, which is related to the success or failure of the cause, the rise and fall of the country, and must be cautious about this. Condensing personal wisdom, interpersonal communication, and even the great wisdom of governing the country, it is further developed into a deep culture that has profoundly affected the Chinese civilization for thousands of years and will surely affect the future [9].

\section{Acknowledgements}

Fund Project: Title of the project: A study on the influence of the social textile and clothing industry in Chinese pre-Qin period on the origins of Huaxia Civilization and social and economic changes (at the provincial level). Question Number: Jilin Province Department of Education "13th Five-Year Plan" social science research project JJKH2017181SK

\section{References}

[1] Nanjing Museum. The Caoshan Mountain Site in Wu County[A]. Cultural Relics Information Series No. 3 [C]. Beijing: Cultural Relics Publishing House, 1980.11

[2] Institute of Archaeology, Chinese Academy of Social Sciences, Shanxi Work Team, etc. The first discovery of copper wares in the ruins of Qintao Temple in Shanxi Province[J]. Archaeology, 1984, (2). 21

[3] Gansu Working Team, Institute of Archaeology, Chinese Academy of Social Sciences. Excavation Report of the Dahezhuang Site in Yongjing, Gansu[J]. Journal of Archaeology, 1974, (2).35

[4] Zhejiang Provincial Cultural Relics Management Committee. The First and Second Excavation Reports of Qianshanjing Site in Wuxing[J]. Acta Archaeological Journal,1960, (2).85

[5] Museum of Hebei Province et al. Shang Dynasty Taixi Village Shang Dynasty Site[M]. Beijing: Cultural Relics Publishing House, 1977:60

[6] Changsha Institute of Archaeology, Chinese Academy of Sciences. Excavation Report [M]. Beijing: Science Press, 1957:63-65.

[7] Northwestern Normal University Institute of Botany, et al. Peony and marijuana excavated from the Linjia Majiayao Cultural Site in Dongxiang, Gansu[J]. Archaeology, 1984, (7).78

[8] Shanghai Textile Science Research Institute, etc. Study on Unearthed Textiles from No. 1 Cemetery of Mawangdui in Changsha[M]. Beijing: Cultural Relic Publishing House, 1990.66

[9] Zhejiang Provincial Cultural Relics Management Committee. First and second excavation report of the Qianshanjing site in Wuxing[J]. Archaeology Journal, 1960, (2).456

[10] Liao Jiangbo, Yang Xiaoming. Research on degumming phenomenon and technology of ramie fiber in Xiabu[J]. Journal of Wuhan Textile University 2016.04.17

[11] Song Jinying, Wang Wei. The Aesthetic Orientation of Hemp Fabrics in Pre-Qin Qi State Dresses[J]. Bulletinics Journal,2016, (4).48 